\title{
UMA PROPOSTA DE PLANEJAMENTO DE PRODUÇÃO VINCULADA A MARGEM DE LUCRO DOS PRODUTOS MANUFATURADOS
}

\section{A PRODUCTION PLANNING PROPOSAL LINKED TO THE PROFIT MARGIN OF MANUFACTURED PRODUCTS}

\author{
Fernanda Romanzini* E-mail feromanzini@outlook.com \\ José Luis Duarte Ribeiro* E-mail: ribeiro@producao.ufrgs.br \\ *Universidade Federal do Rio Grande do Sul (UFRGS), Porto Alegre, RS
}

Resumo: Para alcançar competitividade, as empresas precisam manter seus planos de produção atualizados visando responder com agilidade às demandas do mercado. Neste artigo, é desenvolvido um novo método de planejamento da produção que visa atender às demandas de mercado considerando a margem de lucro dos diferentes produtos. Este resultado é obtido através da combinação de estimativa de vendas, produção programada e margem de lucro dos produtos envolvidos. A aplicação do algoritmo, em uma empresa de manufatura que fornece produtos ao mercado da construção civil, revelou um ganho de $21 \%$ no resultado global em relação ao atual método de planejamento de produção utilizado por esta, comprovando a validade do novo modelo proposto.

Palavras Chaves: Planejamento de produção. Previsão de demanda. Análise de valor agregado.

Abstract: To remain competitive, companies need to keep their productions plans updated to meet market demands. In this paper, we developed a new method for production planning which meets market demands and also considers profit of the different products being produced. This result is achieved through a combination of variables: estimated sales, scheduled production and profit. The implementation of the algorithm in a manufacturing company which provides products to the construction market showed a profit of $21 \%$ in the overall result from the current planning method used by this production, proving the new proposed model.

Keywords: Production planning. Forecasting. Added value analysis.

\section{INTRODUÇÃO}

Atualmente, as empresas atuam em um sistema competitivo, no qual é preciso atentar para as variações do mercado. Estas variações podem ocasionar mudanças favoráveis ou prejudiciais às organizações, sendo que o impacto irá depender do nível de planejamento que a empresa aplica. Assim, é visível a importância do planejamento nas organizações, o qual favorece a tomada de decisão com maior segurança e precisão. Nesse sentido, conforme realça Cezarino (2007), o plano de produção, para empresas de manufatura, assume papel fundamental no processo de planejamento e construção de metas estratégicas. 
Em organizações de médio porte, há dificuldades para implantar e manter o planejamento da produção. As informações disponíveis, muitas vezes desordenadas ou imprecisas, são insuficientes para o estabelecimento de planos de produção, como também a manutenção destes. Porém, o planejamento da produção é essencial para orientar o caminho da organização, integrando o setor comercial e o setor de produção. O propósito básico do Planejamento da Produção, segundo Slack (2002), é garantir que a produção realize seus produtos ou serviços de forma eficiente e eficaz, atendendo às exigências dos consumidores. Para isso é necessário que os recursos produtivos estejam disponíveis na quantidade necessária, no momento adequado e dentro dos parâmetros de qualidade especificados. O planejamento é um assunto de suma importância, uma vez que as empresas convivem com incertezas, aumento da competitividade e novas tecnologias que tornam o ambiente industrial dinâmico e caracterizado pela presença de riscos.

No atual cenário competitivo, as empresas precisam estar em constante análise do ambiente que estão inseridas, pois diversas são as variáveis presentes no ambiente interno e externo das organizações (CHOD e RUDI, 2006; KAPLAN et al., 2010). Logo, faz-se necessário a utilização de planejamento nas diversas esferas empresariais, considerando o conjunto de variáveis que afetam o negócio e visando a maximização do resultado econômico.

Neste artigo, o tema planejamento de produção, no nível tático, é abordado com base nos principais assuntos envolvidos: Planejamento Mestre de Produção (PMP), Previsão de demanda, Capacidade fabril e Análise de Valor Agregado (AVA). Segundo Rubio (2002), o objetivo do planejamento de produção é proporcionar produtos acabados suficientes para o período estabelecido. Atingindo os objetivos de vendas, regularizando os aspectos financeiros e atendendo as restrições da capacidade produtiva. Como afirma Corrêa e Corrêa (2004), o PMP coordena a demanda do mercado com os recursos internos da empresa, e deve ser capaz de programar as taxas adequadas de produtos finais. Para auxiliar na criação do PMP utiliza-se a Previsão de Demanda que, conforme Fusco (2003), é a determinação do que se espera acontecer num determinado tempo futuro que impacta no setor produção, sendo fundamental para o estabelecimento de planos de produção, controle de estoques, bem como para a determinação mais adequada dos produtos a serem produzidos. Para Chase (2006), ao determinar as necessidades referentes à Revista Produção Online, Florianópolis, SC, v. 17, n. 1, p. 200-221, jan./mar. 2017. 
capacidade fabril, é necessário tratar as demandas por linhas de produtos e, assim, diferenciar seu tempo de fabricação, como também os demais dados para possibilitar a sua programação. A utilização da Análise de Valor Agregado (AVA) no ambiente de produção, abordada por Bagherpour et al. (2010), abrange a medição do desempenho da produção e a classificação das tarefas em relação ao valor agregado.

A união destes conceitos possibilita a base necessária para o planejamento da produção. Porém, é raro visualizar isso na prática, uma vez que as organizações, frequentemente, utilizam informações insuficientemente tratadas e de difícil interpretação. Segundo Berry et al. (2005), as empresas estão interessadas em implementar planos de produção e operações, no entanto, continua sendo difícil implantar estes sistemas, uma vez que a integração dos elementos mencionados é complexa.

O objetivo principal deste artigo é desenvolver um método de aplicação do planejamento de produção, baseado nos elementos mencionados acima. Considerando a importância deste método e sua necessidade, busca-se desenvolver procedimentos que permitam realizar a implantação de forma simples e ordenada. Este método pode auxiliar as empresas a reagirem adequadamente às flutuações do mercado, impedindo que as mesmas impactem de forma negativa no seu desempenho.

Adicionalmente, o método proposto pode proporcionar maior integração entre as áreas das organizações, principalmente os setores de vendas (comercial), produção e financeiro. Através da interação entre esses setores, é possível obter melhores resultados, principalmente quando há um plano comum a ser seguido.

\section{REFERENCIAL TEÓRICO}

\subsection{Planejamento de Produção}

O assunto planejamento de produção é amplamente discutido na literatura. Fernandes et al. (2007) mapearam os principais autores e suas respectivas classificações diante dos requisitos avaliados. Similarmente, buscando organizar os assuntos envolvendo planejamento de produção, Mula et al. (2006) apresentam uma revisão da literatura a respeito de modelos para planejamento em ambientes de Revista Produção Online, Florianópolis, SC, v. 17, n. 1, p. 200-221, jan./mar. 2017. 
incerteza. De acordo com esses autores, independente do grau de relação entre os assuntos, todos os trabalhos têm em comum uma formulação de modelagem e geração de planos de produção.

Segundo Carvalho (1998), o objetivo do planejamento é alocar os recursos, conforme sua disponibilidade, às necessidades da produção dos vários produtos, ajustando o custo dos produtos, a forma de operar, a quantidade e o valor do produto final, dentre outros parâmetros de produção. A fim de buscar soluções integráveis para estas atividades, divide-se o planejamento nos níveis estratégicos, tático e operacional, conforme representado na figura 1.

Figura 1 - Hierarquia do Planejamento

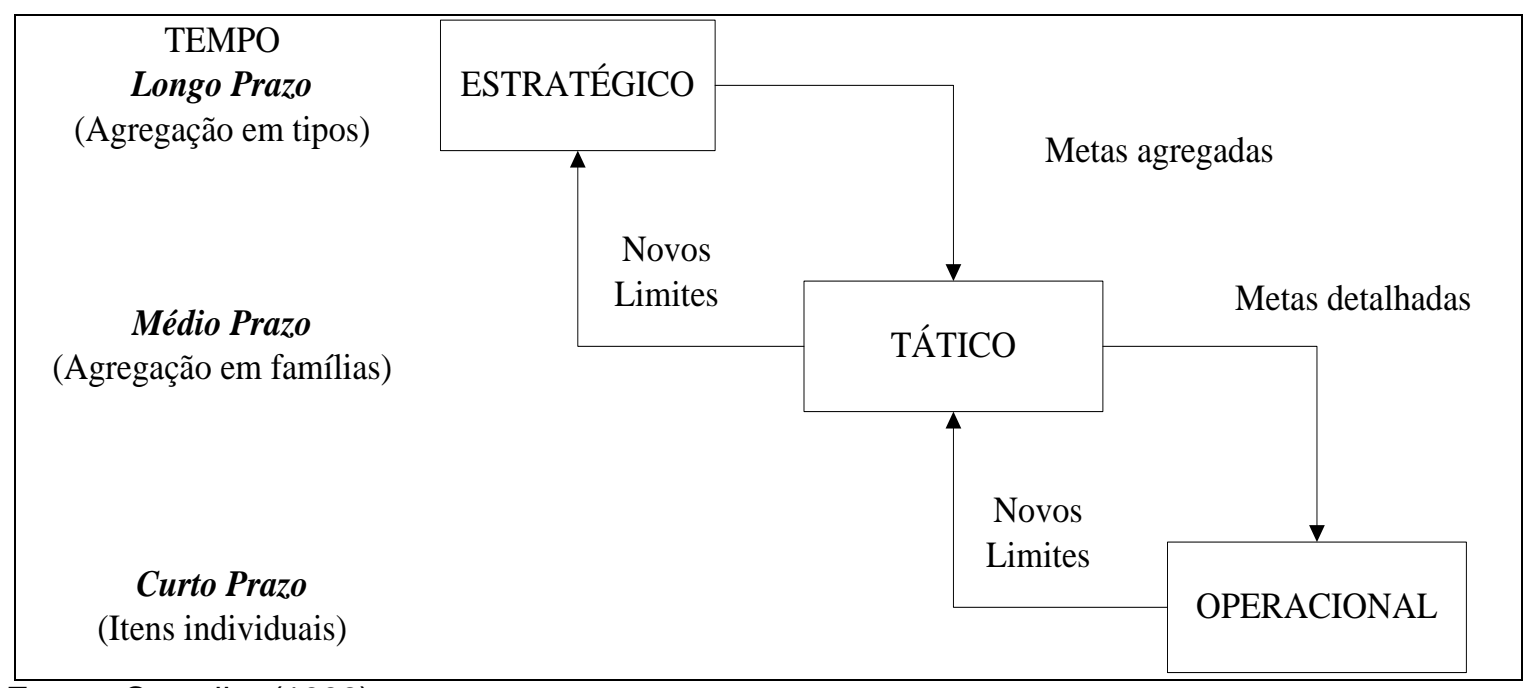

Fonte: Carvalho (1998)

O processo de planejamento de produção pode integrar, além das áreas de produção e comercial, a área contábil (ERDIRIK-DOGAN e GROSSMANN, 2008). Quando isso é feito, as empresas podem realizar seus planos tanto no nível agregado quanto tático, incluindo informações sobre os custos gerais, possibilitando incluir estratégias de maximização dos resultados (KANNEGIESSER et al.,2009).

A hierarquia do planejamento de produção é discutida, também, por Bonney (2000), Bremer (2000) e Silva Filho (2000), os quais têm em comum as seguintes definições sobre as funções de cada nível.

O planejamento no nível estratégico tem como finalidade gerar o plano agregado de produção o qual se baseia nas previsões de demanda em longo prazo. O horizonte de planejamento deste nível é de um ou mais anos. Logo, as informações

Revista Produção Online, Florianópolis, SC, v. 17, n. 1, p. 200-221, jan./mar. 2017. 
sobre o processo, tais como, demanda, capacidade de produção, níveis de mão de obra, estoque, etc., são tratadas de forma agregada, na qual o problema é considerado como alto grau de incerteza.

O nível tático é responsável por desagregar as regras do nível estratégico. Conforme a disponibilidade de recursos, tempo e produtos adequados às políticas ótimas estabelecidas, estas contemplam as informações sobre máquinas, matériaprima, mão-de-obra, etc. O horizonte de planejamento neste nível é de seis meses a um ano. A atividade desenvolvida neste nível é o Plano Mestre de Produção. Logo, as informações são tratadas em um grau de incerteza reduzido.

O planejamento operacional pode ser denominado como programação da produção. A meta deste nível é a execução ótima do plano gerado nos níveis anteriores. Tratando das informações individualmente, tais como: alocação de máquina, operadores, auxiliares, quantidade de matéria prima, tempos de ciclo, etc. Nesta etapa, as atividades são claras e com alto grau de certeza nas informações. $O$ horizonte de planejamento é variável, dependendo do produto realizado pode ser diário, semanal ou mensal, detalhes que não serão explorado neste artigo. O assunto abordado na sequência é o planejamento mestre de produção, que diz respeito ao nível tático.

\subsection{Planejamento Mestre de Produção}

Uma definição para o planejamento mestre de produção (PMP), citado com frequência em trabalhos relacionados ao assunto, é dada pela American Production and Inventory Control Society (APICS) apud Vieira (2009), que afirma que um PMP é uma declaração do que a empresa espera fabricar. Pode-se dizer que é uma série de decisões que orientam o planejamento das necessidades materiais (MRP).

O PMP representa o que a empresa pretende produzir, expresso em termos de configuração, quantidades e datas específicas. O PMP baseia-se no planejamento estratégico da empresa, na previsão de vendas, na pendência de pedidos, na disponibilidade de material, capacidade fabril, políticas gerenciais e objetivos. Após ter realizado todas estas interações, tem-se como resultado as informações de estoque disponível, capacidade utilizada, tempo de processamento. O horizonte de planejamento do PMP é de médio prazo. Este é responsável por transformar o planejamento agregado (tratamento das linhas de produtos) em um plano operacional Revista Produção Online, Florianópolis, SC, v. 17, n. 1, p. 200-221, jan./mar. 2017. 
(tratamento dos itens). Por isso depende da filosofia de gestão da produção adotada pela empresa (IP, 2000; TUBINO, 1997; CORRÊA, 2004).

Segundo Corrêa (2004), o PMP coordena a demanda do mercado com os recursos internos da empresa, capaz de programar as taxas adequadas de produtos finais, principalmente os que possuem demanda independente. Porém o seu mau uso pode pôr a perder as vantagens estabelecidas num bom plano de vendas e produção. Ainda conforme esse autor, ao se preparar um PMP, deve-se levar em consideração algumas questões, tais como:

a) O intervalo de tempo e o horizonte a planejar. Este planejamento trabalha em duas dimensões: determinação da variável de tempo para cada intervalo do plano, e a amplitude, ou horizonte, que possui objetivos diferenciados, um nível firme de horizonte curto, outro com horizonte longo sujeito a alterações;

b) O tratamento diferenciado entre os produtos para estoque e os produtos sob encomendas.

A figura 2 ilustra esses comentários. A parte firme está associada a certeza de demanda e ao lead time do produto. Já na faixa de previsões, se utiliza um PMP flexível.

Em uma abordagem mais recente sobre planejamento da produção visando lucros a longo e médio prazos os níveis de produção, em cenários nos quais os equipamentos sofrem paradas rotineiras, são estabelecidos em relação aos limites de estoque. Uma das conclusões deste estudo é desligar os equipamentos quando os estoques estiverem em níveis acima do limite, e juntamente com esta ação os preços de venda também devem ser baixos. Logo a definição dos parâmetros a serem utilizados como limites é o grande ganho deste estudo (XIUTIAN et al., 2014). 
Figura 2 - Dinâmica do PMP.

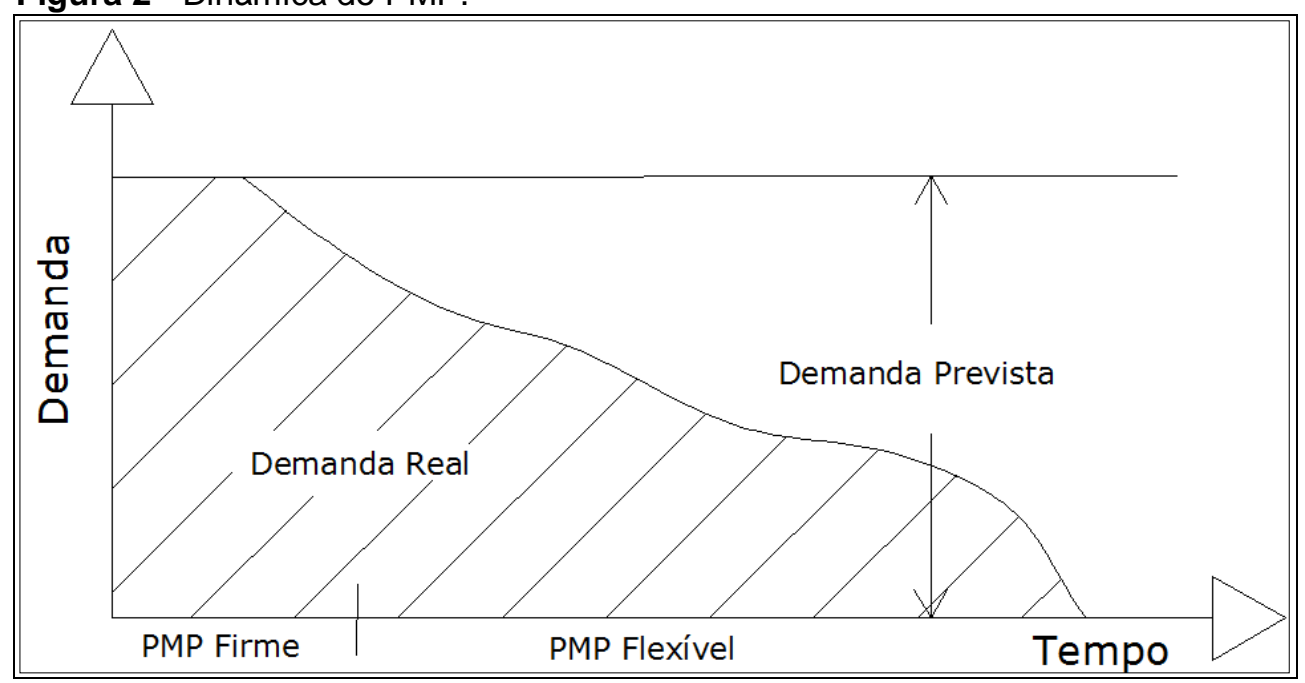

Fonte: Tubino (1997)

\subsection{Planejamento da Capacidade Produtiva}

Ao se determinar o planejamento da capacidade produtiva, é importante ressaltar que a empresa só poderá planejar dentro dos limites produtivos de suas máquinas. Assim, é fundamental que o administrador conheça o potencial dos recursos de sua organização (ROCHA, 1996).

Para compreender o planejamento da capacidade, Corrêa (2001) cita como sendo a atividade que deve ser desenvolvida paralela ao planejamento de materiais. Também ressalta a importância da previsão da capacidade fabril para que não haja deterioração do nível de serviço aos clientes e aumento de estoques em processo, além de gerar frustrações, uma vez que pode não haver possibilidade de atender o programado se a capacidade for superdimensionada. Por outro lado, excesso desnecessário de capacidade representa custos adicionais, com os quais, num ambiente competitivo, nenhuma empresa pode dar-se o luxo de arcar.

Segundo Chase (2006), ao determinar as necessidades de capacidade, é necessário tratar as demandas por linhas de produtos, diferenciando seu tempo de fabricação, como também os demais dados específicos de cada linha, para possibilitar a melhor programação.

A análise da capacidade produtiva no planejamento da produção tem caráter exploratório, possibilitando decisões em longo prazo que só são possíveis se planejadas e implantadas antecipadamente. Essas decisões podem ser mudança nas instalações físicas, compra de equipamentos, inclusão de um novo turno de trabalho,

Revista Produção Online, Florianópolis, SC, v. 17, n. 1, p. 200-221, jan./mar. 2017. 
admissão e treinamentos de mão de obra, contratos de fornecimento e terceirizações (TUBINO, 1997).

Para Rubio (2002), as características que influenciam a função do planejamento da capacidade de máquina estão relacionadas com a disponibilidade e flexibilidade das instalações e equipamentos. Três fatores influenciam nesta função:

a) Se a empresa for pouco flexível, mais detalhadas e formais serão as funções do planejamento da capacidade. Para isso deve haver a flexibilidade da capacidade em curto prazo;

b) Se houver grande número de centros de trabalhos, as funções do planejamento da capacidade deverão ser detalhadas e formais. Isso acontece, pois há maior dificuldade de planejar com altas quantidades de equipamentos;

c) Quanto menos estável a demanda, mais detalhadas as funções do planejamento da capacidade. Ou seja, a estabilidade da demanda também influencia no planejamento.

Chia (2016) propõe um modelo que objetiva auxiliar gestores a resolver o dilema de capacidade. O estudo emprega a abordagem pesar Minimax Regret (MMR) e a técnica de programação Estocástica para enfrentar variações alvo, causada por flutuações de demanda. Como conclusão tem-se que o planejamento da capacidade integrando as perspectivas da economia e gestão da produção ajudam as empresas a alcançarem seus objetivos e evitar rupturas de capacidade.

\subsection{Previsão de Demanda}

Para Pellegrini (2001), um sistema de previsão de demanda deve estabelecer relações entre previsões feitas pelas diferentes áreas de gerenciamento. Logo, criase uma relação de dependência entre as áreas, que devem estar em sintonia, pois isso define o sucesso na implantação do sistema de previsão de demanda. Por exemplo, um erro na projeção de vendas pode trazer uma série de prejuízos a todas as demais áreas da organização.

Ainda conforme esse autor, as técnicas de previsão de demanda quantitativas oscilam consideravelmente, pois foram desenvolvidas com diferentes propósitos. 
Cada uma possui características próprias, grau de acuracidade e custos, as quais devem ser consideradas na escolha de um método necessário.

De modo geral, as diversas técnicas de previsão de demanda podem ser classificadas em: qualitativa, análise de séries temporais e modelos causais (CHASE, 2006). Neste trabalho, será abordada a análise de séries temporais, a qual se baseia em dados passados, ou seja, utiliza históricos de demanda para continuar gerando a demanda no futuro. Conforme Chase (2006), as séries temporais podem ser divididas em média móvel simples, média ponderada exponencial, análise de regressão e projeção de tendência.

Em estudo mais recentes $\mathrm{O}$ administrador do sistema deve determinar o preço de venda e o nível de produção em cada ponto de decisão. Em primeiro lugar, mostrar que a política de produção e preços ótima é um controle de limite que é caracterizado por três parâmetros de limites sob a longo prazo tanto o lucro e com desconto critérios de longo prazo de lucro média. Em seguida, estabelecer as relações estruturais entre os três parâmetros que limiar de produção está desligado quando o estoque está acima do limite, e o preço de venda ideal que deve ser baixo quando o estoque está acima do limite sob o cenário onde a máquina está baixo ou para cima.

\subsection{Análise de Valor Agregado (AVA)}

Dividem-se as operações em dois tipos: as que agregam valor, sendo estas que realmente transformam a matéria-prima, modificando a forma, e as que não agregam valor, como por exemplo, as movimentações e transportes, ou seja, as perdas do processo.

A Análise de Valor Agregado (AVA) tem sua aplicação bastante difundida na área de gerenciamento de projetos. Vandevoorde (2005) e Vitner et al. (2006) tratam o assunto como gerenciamento de valor agregado (GVA), que significa um sistema de gestão na área de projetos que integra desempenho de custos, cronograma e técnica. Além de permitir o cálculo de indices de desempenho, previsão de custos e duração do cronograma. Esta análise é responsável por fornecer os primeiros indícios do desempenho do projeto, e em seguida aponta as ações corretivas necessárias.

Outra abordagem, relatada por Severiano (1997), diz que o valor agregado de um produto representa a diferença entre o valor do produto final e o valor do material. 
Logo, pode-se dizer que a análise de valor agregado persiste na forma com que o produto é transformado. Esta transformação acarreta custos de processamento que posteriormente devem ser embutidos no preço final do produto.

Genericamente, a análise de valor agregado é uma simples comparação entre os gastos obtidos e o que se planejava gastar (OLIVEIRA, 2003).

\section{MÉTODO PROPOSTO}

Quanto à natureza, esta pesquisa classifica-se como aplicada, na medida em que é orientada à solução de um problema prático, envolvendo a integração de aspectos associados a valor agregado, programação da produção e capacidade fabril. Quanto à abordagem, a pesquisa é essencialmente quantitativa, pois serão utilizados conceitos matemáticos e estatísticos na solução do problema. Quanto aos objetivos, classifica-se, predominantemente, como pesquisa explicativa, onde a ênfase é a construção de um modelo de previsão e programação de produção. Por fim, quanto aos procedimentos, trata-se de uma pesquisa-ação, uma vez que os autores estiveram diretamente envolvidos na pesquisa e implementação da solução de um problema prático.

A construção do modelo se deu pela necessidade de uma solução prática para um problema enfrentado por uma companhia específica, mas que é facilmente encontrado em outras empresas de manufatura. A partir da análise realizada, optouse por utilizar as ferramentas mais simples para que a solução fosse facilmente aplicada. Logo, a estruturação do modelo foi baseada na literatura, porém com o foco na aplicabilidade e realidade da empresa em estudo.

O trabalho é realizado em quatro etapas, que envolvem: (I) coleta e organização de dados, (ii) estabelecimento do algoritmo que agregue previsão de demanda, análise de valor agregado, análise da capacidade fabril; (iii) estabelecimento do plano mestre de produção, considerando os resultados anteriores; e (iv) teste desses algoritmos no cenário real. A figura 3 apresenta as fases que compõem o método proposto e as etapas a serem seguidas. 
Figura 3 - Fases e etapas do método proposto.

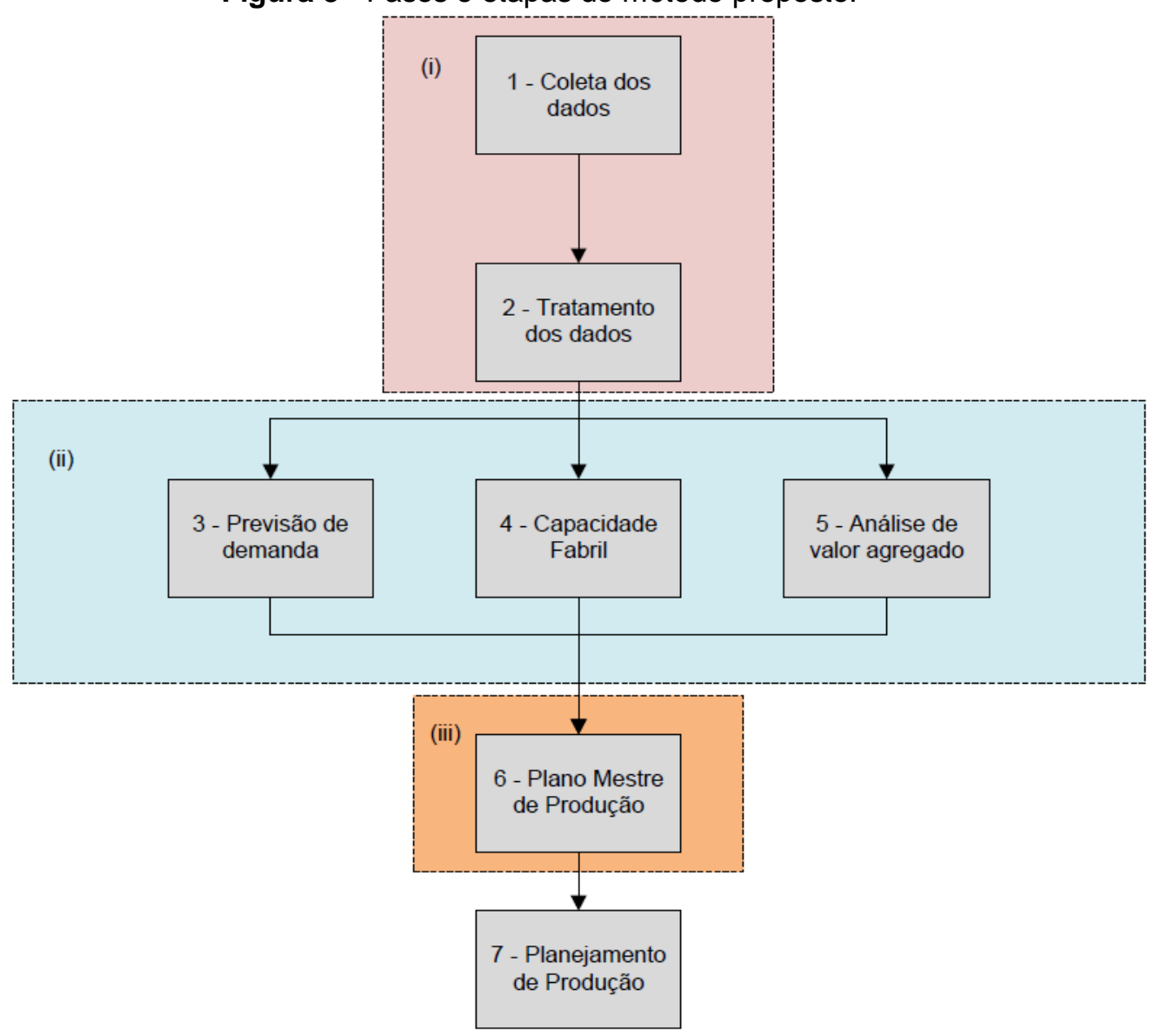

Fonte: Elaborado pelos autores.

\subsection{Coleta e tratamento dos dados}

Para que os algoritmos, que compõem o método proposto, possam ser aplicados, é necessária uma base de dados com as informações dos itens vendidos nos últimos meses. Para contemplar variações sazonais, essa base de dados deve contemplar um período mínimo de 12 meses, sendo recomendado um histórico de pelo menos 36 meses. Apoiado na base de dados é possível analisar o comportamento da demanda nos últimos meses, que poderá ser refletido para os próximos meses. Existindo necessidade, os dados devem ser analisados por grupo de estoque, famílias comerciais ou itens individuais.

É importante salientar que a importância do tratamento das bases de dados abordadas em períodos mensais, excluindo-se os dados atípicos, tais como: itens descontinuados no período, itens especiais (produção com características exclusivas e destinadas a pedidos especiais), como também valores espúrios decorrentes de promoções esporádicas de vendas.

Revista Produção Online, Florianópolis, SC, v. 17, n. 1, p. 200-221, jan./mar. 2017. 
Paralelamente, também devem ser estruturadas bases de dados com informações referentes à listagem dos equipamentos disponíveis, informando suas respectivas capacidades e restrições, para que na sequência os produtos possam ser alocados nestes equipamentos.

Outra informação pertinente é o valor agregado por produto, que neste estudo é representado pela margem de lucro. Sendo assim, adota-se a margem de lucro como índice do valor agregado para todos os itens tratados neste modelo.

\subsection{Previsão de demanda, análise de valor agregado e análise da capacidade fabril}

Em cada situação deve ser utilizado o método de previsão de demanda apropriado para tratar os dados. Entre os métodos de previsão de demanda utilizados com maior frequência, destacam-se: (I) métodos causais onde a demanda dos itens é relacionada à variáveis internas ou externas à empresa; e (ii) as séries temporais, que exigem apenas o conhecimento de valores passados da demanda. Neste caso, utilizou-se um método de séries temporais, onde a previsão de demanda apoia-se nos dados históricos das vendas.

Ao obter o valor da previsão de demanda para os meses futuros, deve-se acompanhar as demandas reais durante um período mínimo de seis meses consecutivos, a fim de obter o valor do erro de previsão, considerado como a variabilidade da demanda no período. Estas informações fazem parte da distribuição de probabilidade da demanda $(f(x))$, utilizada para obter as vendas esperadas para o período, valor que é calculado através da equação 1.

$$
\widehat{V}_{l}=\int_{0}^{P_{i}} x f_{i}(x) d x+P \int_{P_{i}}^{\infty} f_{i}(x) d x
$$

Onde $V_{i}$ corresponde ao volume esperado das vendas para o produto i, $\mathrm{x}$ é a demanda, $f(x)$ é a distribuição de probabilidade da demanda, Pi corresponde ao valor programado de produção.

O valor programado de produção $\left(P_{i}\right)$ é obtido através de programação matemática, visando otimizar a capacidade produtiva e a maximização da margem de lucro do sistema. No algoritmo desenvolvido são informados os valores de margem de lucro $\left(M C_{i}\right)$ de cada item e as respectivas restrições dos equipamentos de 
produção. Como o objetivo é maximizar o resultado, o modelo visa produzir os itens que possuem maior valor agregado, ou seja, maior margem de lucro, neste caso. Logo, se algum item apresentar margem negativa, este tem sua produção minimizada, priorizando-se a produção dos itens que contemplam margem positiva.

A listagem dos equipamentos disponíveis e suas respectivas capacidades e restrições devem ser levantadas ainda na etapa anterior. No entanto, o compartilhamento dos equipamentos, ou seja, a alocação dos itens nos equipamentos disponíveis, respeitando as restrições e capacidades devem ser realizadas nesta etapa para que seja possível realizar o cálculo da capacidade disponível.

Como ( $I$ ) o objetivo da produção programada é maximizar o uso da capacidade produtiva e (ii) o valor esperado de vendas depende da previsão de demanda no período, é possível que o sistema gere estoque dos itens estudados. Conforme, apresentado na equação 2.

$$
\widehat{E}_{l}=P_{i}-\widehat{V}_{l}
$$

Onde $E_{i}$ corresponde ao valor esperado do Estoque para o produto $i$, tendo em vista o valor esperado das vendas e o valor programado da produção.

O cálculo do resultado econômico, a ser maximizado, está representado pela equação 3.

$$
\widehat{R}_{\iota}=\widehat{V}_{l} \times M C_{i}-\widehat{E}_{l} \times C E_{i}
$$

Onde $\hat{R}_{i}$ corresponde ao valor esperado do resultado econômico associado ao produto $i$, considerando a margem de lucro $\left(M C_{i}\right)$ e os custos de estocagem $\left(C E_{i}\right)$, valores próprios de cada produto analisado. Os custos de estocagem têm papel importante no algoritmo, pois nesta variável estão inclusos os custos de deslocamento, armazenagem, eventuais perdas físicas e dinheiro sem movimentação. Além disso, essa variável envolve uma restrição para que o sistema não resulte em uma quantidade excessiva de estoques.

\subsection{Plano Mestre de Produção e Planejamento de Produção}

A partir das etapas apresentadas acima, busca-se maximizar o Resultado Econômico Global $\left(R_{G}\right)$. Conforme mencionado, para que se possa planejar a produção, é preciso considerar a capacidade fabril, buscando determinar uma solução ótima que alcance a melhor utilização dos equipamentos disponíveis. Logo, a

Revista Produção Online, Florianópolis, SC, v. 17, n. 1, p. 200-221, jan./mar. 2017. 
maximização do resultado econômico dependerá predominantemente das vendas esperadas para o período, que por sua vez, dependem da previsão de demanda e da produção programada, a qual está atrelada aos itens que apresentam maior valor agregado, no entanto, sujeitos às restrições referentes à capacidade fabril.

Através da reunião dessas informações, é possível estruturar o plano agregado de produção que trata os resultados por linha ou família de produtos. Para executar o plano mestre de produção é necessário fazer o desdobramento das famílias de produtos em seus respectivos itens.

\subsection{Teste dos algoritmos implementados}

Os algoritmos implementados, que envolvem: (I) a previsão de demanda e (ii) o planejamento da produção, considerando a capacidade fabril, plano mestre de produção, análise de valor agregado, foram usados em um caso prático envolvendo dez famílias de produtos e sete equipamentos disponíveis para a fabricação destas. Através da implementação dos algoritmos é possível explicar a utilidade e, consequentemente, o desempenho do algoritmo desenvolvido.

\section{RESULTADOS}

\subsection{Aplicação da metodologia}

Nesta seção são apresentados os resultados da aplicação do método proposto em uma empresa fabricante de materiais em PVC para a construção civil. Os produtos contemplados são usados nos acabamentos de residências e lojas. A empresa possui atuação nacional na comercialização de seus produtos. Com diversas unidades produtivas situadas ao longo do território nacional.

Ao aplicar o método descrito no capítulo anterior, foram obtidos resultados satisfatórios em relação aos objetivos gerais do estudo. É importante salientar que a estrutura do modelo proposto apresenta uma nova forma de observar os diversos ângulos que envolvem o planejamento de produção. A principal ferramenta utilizada é a análise de valor agregado, que neste artigo fora representado pela margem de lucro das linhas de produtos, possibilitando a comparação destes.

Revista Produção Online, Florianópolis, SC, v. 17, n. 1, p. 200-221, jan./mar. 2017. 
Pode-se dizer que o estabelecimento do Plano Mestre de Produção seguindo os algoritmos propostos representa uma abordagem econômica, que explicita a busca de uma solução que maximize o resultado econômico.

A aplicação do método ocorreu de forma simples, com a utilização de algoritmos programados em planilhas eletrônicas. Os algoritmos envolvem diversos parâmetros de simples determinação, tais como: venda nos últimos meses, custo de estocagem, margem de lucro por produto, capacidade dos equipamentos instalados.

O algoritmo proposto considera os dados históricos de vendas, os quais permitiram obter uma previsão e esta foi comparada com os dados reais de vendas por um período de sete meses. A previsão de vendas foi feita utilizando um modelo que incorpora as parcelas usuais correspondentes a média, sazonalidade e tendência conhecido como suavização dupla de Holt-Winters. Os métodos estatísticos de suavização exponencial Holt-Winters são amplamente utilizados para fornecer previsões de curto prazo para os dados de vendas e os níveis de demanda, devido a sua simplicidade, baixo custo de operação, boa precisão, capacidade de ajustamento automático e rápido as mudanças na série temporal em análise.

Comparando valores previstos e observados, foi constatado um erro absoluto de previsão de $41 \%$, o que confirma grande variabilidade nas vendas mês a mês. Ao interpretar estes valores, nota-se um percentual de erro mais elevados nos itens que possuem margem de lucro próxima ou inferior a zero. Isso significa que o método proposto neste artigo está sendo eficaz, uma vez que as ações para diminuir a venda e consequentemente a produção dos itens que possuem baixo valor agregado foram realizadas.

A fim de, simplificar a solução do algoritmo quanto a capacidade produtiva, os itens foram alocados em grupos conforme a similaridade ou compartilhamento dos equipamentos utilizados para fabricação, conforme apresentado no quadro 1. 
Quadro 1 - Agrupamento dos itens por compartilhamento de equipamentos.

\begin{tabular}{|ccc|}
\hline \multirow{2}{*}{ Grupo } & Descrição Produto & Equipamentos compartilhados \\
\hline \multirow{3}{*}{1} & ITEM A & $112,113,114,116$ \\
\cline { 2 - 3 } & ITEM B & $112,113,114,116$ \\
\cline { 2 - 3 } & ITEM C & $112,113,114,116$ \\
\hline \multirow{2}{*}{2} & ITEM D & $112,113,114,116$ \\
\hline \multirow{2}{*}{3} & ITEM E & 109 \\
\hline \multirow{2}{*}{4} & ITEM F & 109 \\
\hline & ITEM G & 103 \\
\hline & ITEM H & 103 \\
\hline
\end{tabular}

Fonte: elaborado pelos autores

Ao realizar o agrupamento dos itens que possuem processos semelhantes é possível diminuir o número de variáveis que interagem no algoritmo, pois evita a realização do cruzamento de informações desnecessárias. Como é possível observar no quadro 1 , o grupo 1 foi criado através da união de item que tinham por características o compartilhamento dos mesmos equipamentos e tempos de processamento semelhantes. O mesmo acontece para o grupo 2 e os demais. Este procedimento foi utilizado para reduzir o número de iterações no algoritmo, no entanto sabe-se que em outros casos e aplicações esta etapa pode não ser utilizada.

O segundo algoritmo tem como objetivo calcular o valor esperado de vendas, que é obtido através da resolução da integral apresentada na equação 1.

Para determinação da produção programada no período, utilizou-se as rotinas de programação linear implementadas em planilhas eletrônicas que, basicamente, cruzaram a capacidade fabril dos equipamentos disponíveis, e priorizam a produção dos itens que apresentam melhor margem de lucro. A tabela 1 apresenta as restrições de capacidade, valor agregado, representado pela margem de lucro do item e quantidade programada de produção, ligada a maximização da capacidade produtiva. 
Tabela 1 - Cálculo da produção programada, envolvendo as restrições de capacidade fabril e valor agregado.

\begin{tabular}{|c|c|c|c|c|c|c|}
\hline Grupo & $\begin{array}{l}\text { Descriçã } \\
\text { o Item }\end{array}$ & $\begin{array}{c}\text { Produção } \\
\text { Programada (Kg) }\end{array}$ & $\mathrm{Ma}$ & $\begin{array}{l}\text { em de } \\
\text { cro }\end{array}$ & $\begin{array}{l}\text { Capac. por } \\
\text { item (Kg) }\end{array}$ & $\begin{array}{l}\text { Capac. Equip. } \\
(\mathrm{Kg})\end{array}$ \\
\hline \multirow{5}{*}{1} & Item A & 100.000 & $\mathrm{R} \$$ & 1,12 & 100.000 & \multirow{5}{*}{450.000} \\
\hline & Item B & 100.000 & $\mathrm{R} \$$ & 0,90 & 100.000 & \\
\hline & Item C & 200.000 & $\mathrm{R} \$$ & 0,10 & 200.000 & \\
\hline & Item D & - & $-R \$$ & 0,22 & 100.000 & \\
\hline & Total & 400.000 & & & & \\
\hline \multirow{3}{*}{2} & Item E & 80.000 & $\mathrm{R} \$$ & 10,35 & 80.000 & \multirow{3}{*}{100.000} \\
\hline & Item F & 20.000 & $\mathrm{R} \$$ & 1,19 & 100.000 & \\
\hline & Total & 100.000 & & & & \\
\hline \multirow{3}{*}{3} & Item G & 10.000 & $\mathrm{R} \$$ & 13,03 & 30.000 & \multirow{3}{*}{30.000} \\
\hline & Item H & 20.000 & $\mathrm{R} \$$ & 14,70 & 30.000 & \\
\hline & Total & 30.000 & & & & \\
\hline \multirow{3}{*}{4} & Item I & 10.000 & $\mathrm{R} \$$ & 18,10 & 10.000 & \multirow{3}{*}{120.000} \\
\hline & Item J & 110.000 & $\mathrm{R} \$$ & 2,44 & 120.000 & \\
\hline & Total & 120.000 & & & & \\
\hline
\end{tabular}

Fonte: Elaborado pelos autores.

\subsection{Resultados da aplicação do método}

A fim de comprovar a eficácia do método apresentado, este será comparado com outras duas situações, onde a quantidade a ser produzida varia conforme os critérios aplicados. A primeira situação converte a previsão de vendas (baseado somente nos históricos) na quantidade a ser produzida, sem considerar o valor agregado dos produtos. A segunda é baseada no conhecimento empírico dos programadores de produção da empresa, ou seja, sem nenhum método formal. E, por último, a aplicação da metodologia apresentada neste estudo. O resultado em cada uma das situações é obtido através de simulações. Nestas existem itens em que a margem de lucro é negativa, proporcionando resultados negativos.

O objetivo é otimizar o valor do resultado mensal, representado pela expressão 4:

$$
\left.\widehat{R_{A, J}}=\widehat{V_{A, J}} \times M C_{A, J}-\widehat{E_{A, J}} \times C E_{A, J} \quad \text { (eq. } 4\right)
$$

Conforme apresentado na tabela 2, a quantidade produzida programada foi baseada somente na previsão de vendas, baseada no histórico dos últimos 3 anos, não considerando o valor agregado dos produtos. 
Tabela 2 - Método de programação de produção baseado somente no histórico de vendas.

\begin{tabular}{lccrrrrr}
\hline Item & $\begin{array}{c}\text { Produção } \\
\text { sugerida } \\
(\mathrm{Kg})\end{array}$ & $\begin{array}{c}\text { Qtde real } \\
\text { vendida no } \\
\text { período }(\mathrm{Kg})\end{array}$ & $\begin{array}{c}\text { Qtd estocada } \\
(\mathrm{Kg})\end{array}$ & $\begin{array}{c}\text { Custo estocagem } \\
(\mathrm{R} \$ / \mathrm{Kg})\end{array}$ & Resultado Simulado \\
\hline Item A & 28.804 & 40.469 & - & $\mathrm{R} \$$ & 0,95 & $\mathrm{R} \$$ & $32.299,99$ \\
Item B & 10.738 & 13.153 & - & $\mathrm{R} \$$ & 0,95 & $\mathrm{R} \$$ & $9.711,16$ \\
Item C & 173.632 & 124.342 & $49.290,78$ & $\mathrm{R} \$$ & 0,95 & $-\mathrm{R} \$$ & $34.392,08$ \\
Item D & 1.139 & 1.234 & - & $\mathrm{R} \$$ & 0,95 & $-\mathrm{R} \$$ & 250,63 \\
Item E & 59.789 & 43.119 & $16.670,22$ & $\mathrm{R} \$$ & 0,95 & $\mathrm{R} \$$ & $430.569,33$ \\
Item F & 7.426 & 13.632 & - & $\mathrm{R} \$$ & 0,95 & $\mathrm{R} \$$ & $8.810,96$ \\
Item G & 2.494 & 5.707 & - & $\mathrm{R} \$$ & 0,95 & $\mathrm{R} \$$ & $32.492,87$ \\
Item H & 1.986 & 2.252 & - & $\mathrm{R} \$$ & 0,95 & $\mathrm{R} \$$ & $29.192,19$ \\
Item I & 9.199 & 5.987 & $3.212,07$ & $\mathrm{R} \$$ & 0,95 & $\mathrm{R} \$$ & $105.339,26$ \\
Item J & 82.820 & 57.339 & $25.481,29$ & $\mathrm{R} \$$ & 0,95 & $\mathrm{R} \$$ & $115.424,48$ \\
& & & & & \multicolumn{7}{c}{ TOTAL } & $\mathrm{R} \$$ & $729.197,54$ \\
\hline
\end{tabular}

Fonte: elaborado pelos autores

Na tabela 3, o resultado obtido se dá através de uma programação de produção baseada no conhecimento empírico dos colaboradores da empresa e nas restrições de capacidade fabril.

Tabela 3 - Método de programação de produção baseado no conhecimento empírico dos colaboradores.

\begin{tabular}{|c|c|c|c|c|c|c|c|}
\hline \multirow{2}{*}{$\begin{array}{c}\text { Item } \\
\text { Item A }\end{array}$} & \multirow{2}{*}{$\begin{array}{c}\begin{array}{c}\text { Produção } \\
\text { sugerida }(\mathrm{Kg})\end{array} \\
45.000\end{array}$} & \multirow{2}{*}{$\begin{array}{c}\text { Qtde real vendida } \\
\text { no período }(\mathrm{Kg})\end{array}$} & \multirow{2}{*}{$\begin{array}{c}\begin{array}{c}\text { Qtd } \\
\text { estocada } \\
(\mathrm{Kg})\end{array} \\
4.531\end{array}$} & \multicolumn{2}{|c|}{$\begin{array}{c}\text { Custo } \\
\text { estocagem } \\
\mathrm{R} \$ / \mathrm{Kg} \\
\end{array}$} & \multicolumn{2}{|c|}{ Resultado Simulado } \\
\hline & & & & $\mathrm{R} \$$ & 0,95 & $\mathrm{R} \$$ & $41.076,16$ \\
\hline Item B & 15.000 & 13.153 & 1.847 & $\mathrm{R} \$$ & 0,95 & $\mathrm{R} \$$ & $10.139,75$ \\
\hline Item C & 120.000 & 124.342 & 0 & $\mathrm{R} \$$ & 0,95 & $\mathrm{R} \$$ & $12.000,00$ \\
\hline Item D & 100.000 & 1.234 & 98.766 & $\mathrm{R} \$$ & 0,95 & $-R \$$ & $94.099,35$ \\
\hline Item $\mathrm{E}$ & 60.000 & 43.119 & 16.881 & $\mathrm{R} \$$ & 0,95 & $\mathrm{R} \$$ & $430.369,22$ \\
\hline Item $\mathrm{F}$ & 12.000 & 13.632 & 0 & $\mathrm{R} \$$ & 0,95 & $\mathrm{R} \$$ & $14.238,74$ \\
\hline Item G & 15.000 & 5.707 & 9.293 & $\mathrm{R} \$$ & 0,95 & $\mathrm{R} \$$ & $65.532,46$ \\
\hline Item $\mathrm{H}$ & 4.500 & 2.252 & 2.249 & $\mathrm{R} \$$ & 0,95 & $\mathrm{R} \$$ & $30.960,98$ \\
\hline Item I & 3.466 & 5.987 & 0 & $\mathrm{R} \$$ & 0,95 & $\mathrm{R} \$$ & $62.749,67$ \\
\hline \multirow[t]{2}{*}{ Item J } & 19.000 & 57.339 & 0 & $\mathrm{R} \$$ & 0,95 & $\mathrm{R} \$$ & $46.268,64$ \\
\hline & & & & \multicolumn{2}{|c|}{ TOTAL } & $\mathrm{R} \$$ & $619.236,27$ \\
\hline
\end{tabular}

Fonte: elaborado pelos autores

A tabela 4 corresponde aos resultados alcançados através da metodologia proposta neste estudo, considerando o valor agregado dos produtos. Como é possível observar, este método fornece 0 melhor resultado dentre as três maneiras apresentadas para calcular a produção programada.

Ao obter os resultados apresentados nas tabelas 2 a 4, é possível comprovar a utilidade do método aqui desenvolvido. A tabela 4, que representa a aplicação do Revista Produção Online, Florianópolis, SC, v. 17, n. 1, p. 200-221, jan./mar. 2017. 
modelo utilizando a análise de valor dos produtos, que apresentou o melhor resultado, seguido da aplicação apenas da previsão de demanda, também desenvolvida neste modelo, e por último da programação de forma empírica. Neste último caso, é possível perceber que ocorre uma perda de aproximadamente $R \$ 170.000,00$, ou seja, deixase de ganhar $21 \%$ ao utilizar este método, comparado ao primeiro. Quando aplicada somente a previsão de demanda, a diferença é menor, em torno de $R \$ 60.000,00$, no entanto, ainda representa uma perda de $8 \%$ no resultado global, comparado ao modelo utilizando a análise de valor agregado.

Tabela 4 - Método de programação da produção através da análise do valor agregado.

\begin{tabular}{|c|c|c|c|c|c|c|c|}
\hline \multirow{2}{*}{$\begin{array}{l}\text { Item } \\
\text { Item A }\end{array}$} & \multirow{2}{*}{$\begin{array}{c}\begin{array}{c}\text { Produção } \\
\text { sugerida }(\mathrm{Kg})\end{array} \\
28.839\end{array}$} & \multirow{2}{*}{$\begin{array}{c}\text { Qtde real vendida } \\
\text { no período }(\mathrm{Kg})\end{array}$} & \multirow{2}{*}{$\begin{array}{l}\text { Qtd } \\
\text { estocada } \\
\text { (Kg) }\end{array}$} & \multicolumn{2}{|c|}{$\begin{array}{c}\text { Custo } \\
\text { estocagem } \\
\mathrm{R} \$ / \mathrm{Kg}\end{array}$} & \multicolumn{2}{|c|}{ Resultado Simulado } \\
\hline & & & & $\mathrm{R} \$$ & 0,95 & $\mathrm{R} \$$ & $32.339,26$ \\
\hline Item B & 10.753 & 13.153 & - & $\mathrm{R} \$$ & 0,95 & $\mathrm{R} \$$ & $9.724,59$ \\
\hline Item C & 95.180 & 124.342 & - & $\mathrm{R} \$$ & 0,95 & $\mathrm{R} \$$ & $9.517,98$ \\
\hline Item D & 0 & 1.234 & - & $\mathrm{R} \$$ & 0,95 & $\mathrm{R} \$$ & - \\
\hline Item E & 54.089 & 43.119 & $10.969,43$ & $\mathrm{R} \$$ & 0,95 & $\mathrm{R} \$$ & $435.985,08$ \\
\hline Item F & 7.439 & 13.632 & - & $\mathrm{R} \$$ & 0,95 & $\mathrm{R} \$$ & $8.827,18$ \\
\hline Item G & 2.498 & 5.707 & - & $\mathrm{R} \$$ & 0,95 & $\mathrm{R} \$$ & $32.552,66$ \\
\hline Item $\mathrm{H}$ & 1.989 & 2.252 & - & $\mathrm{R} \$$ & 0,95 & $\mathrm{R} \$$ & $29.240,55$ \\
\hline Item I & 7.222 & 5.987 & $1.235,07$ & $\mathrm{R} \$$ & 0,95 & $\mathrm{R} \$$ & $107.217,41$ \\
\hline \multirow[t]{2}{*}{ Item J } & 74.612 & 57.339 & $17.272,90$ & $\mathrm{R} \$$ & 0,95 & $\mathrm{R} \$$ & $123.222,45$ \\
\hline & & & & \multicolumn{2}{|c|}{ TOTAL } & $\mathrm{R} \$$ & $788.627,15$ \\
\hline
\end{tabular}

Fonte: elaborado pelos autores

\section{CONCLUSÃO}

O Planejamento de Produção é uma atividade importante, pois direciona tipos de produtos e quantidades a serem produzidos, em um determinado período. Esta deve acompanhar as tendências de mercado e buscar constantemente resultados positivos para a organização.

Visando alcançar esses objetivos, este trabalho apresentou um novo método para realização do plano mestre de produção e discutiu sua aplicação, mostrando detalhadamente as etapas a serem seguidas e as variáveis consideradas. A principal característica do método proposto é a integração de previsão de vendas, capacidade fabril e análise de valor agregado. O método se mostrou simples e de fácil aplicação. 
O modelo apresentado neste artigo contempla múltiplos produtos, alocados em múltiplos equipamentos, que por sua vez, são ocupados, em geral, por mais de um produto. É importante salientar que o modelo opera com capacidade finita, uma vez que serve de suporte para empresas reais de manufatura. A demanda é tratada de forma estocástica e dependente do histórico de vendas. A produção é otimizada, a fim de maximizar o lucro do sistema, considerando as limitações de capacidade produtiva.

A aplicação do método atendeu às expectativas. O teste do método proposto comparou o mesmo com duas outras maneiras de programar a produção, sendo estas, quando a produção programada é exatamente igual a previsão de vendas (baseada somente em histórico) e quando não há nenhum método utilizado, apenas utilizando a restrição de capacidade máxima e o conhecimento empírico dos programadores de produção. Comparando os resultados obtidos diante de cada maneira de programação da produção observou-se que o método proposto gerou resultados econômicos superiores.

Por fim, vale mencionar que o trabalho realizado foi de grande importância para a empresa que foi objeto de estudo, uma vez que esta não possui um método para desenvolvimento do Plano Mestre de Produção, que atualmente baseia-se apenas no conhecimento empírico de seus colaborados.

\section{REFERÊNCIAS}

BAGHERPOUR, M.; ZAREEI, A.; NOORI, S.; HEYDARI, M. Designing a control mechanism using earned value analysis: an application to production environment. International Journal Advanced Manufacturing Technology, v. 49, p. 419-429, 2010.

http://dx.doi.org/10.1007/s00170-009-2406-z

BERGAMO, E.; BATTAGLIA, D. Análise de valor e engenharia de valor: uma ferramenta de redução de custos em um projeto. P\&D em Engenharia de Produção, v. 8 (3), p. 102-115, 2010.

BERRY, W. L.; WHYBARK, D. C.; JACOBS, F. R.; VOLLMANN, T. E. Manufacturing Planning and Control Systems for Supply Chain Management, 5 ed. São Paulo: Bookman, 2005.

BONNEY, M. Reflections on Production Planning and Control (PPC). Gestão \& Produção, v. 7 (3), p. 181-207, 2000. http://dx.doi.org/10.1590/S0104-530X2000000300002

BREMER, C. F.; LENZA, R. P. Um Modelo de Gestão de Referência para Gestão da Produção em Sistemas de Produção Assembly To Order - ATO e suas Múltiplas Aplicações. Gestão \& Produção, v. 7 (3), p. 269-282, 2000. http://dx.doi.org/10.1590/S0104$\underline{530 \times 2000000300006}$

Revista Produção Online, Florianópolis, SC, v. 17, n. 1, p. 200-221, jan./mar. 2017. 
CARVALHO, M. F.; SILVA FILHO, O. S.; FERNANDES, C. A. O. O Planejamento da Manufatura - Práticas Industriais e Métodos de Otimização. Gestão \& Produção, v. 5 (1), p. 34-59, 1998. http://dx.doi.org/10.1590/S0104-530X1998000100003

CEZARINO, W.; SILVA FILHO, O. S. Geração de planos de produção via otimização seqüencial sub-ótima. Gestão \& Produção, v. 14 (2), p. 239-252, 2007.

CHASE, R. B; JACOBS, F. R.; AQUILANO, N. J. Administração da produção para a vantagem competitiva, Porto Alegre: Bookman, 2006.

CHIA, Y. E. Most productive scale size versus demand fulfillment: A solution to the capacity dilemma. European Journal of Operational Research, v 248, p. 954-962, 2016. DOI: $\underline{10.2139 / \text { ssrn.2380129 }}$

CHOD, J.; RUDI, N. Strategic investments, trading, and pricing under forecast updating. Management Science, v. 52 (12), p. 1913-1929, 2006. http://dx.doi.org/10.1287/mnsc.1060.0574

CORRÊA, H. L.; CORRÊA, C. A. Administração de produção e operações, manufatura e serviços: uma abordagem estratégica, São Paulo: Atlas, 2004.

CORRÊA, H. L.; GIANESI, I. G. N.; CAON, M. Planejamento, programação e controle da produção: MRPII/ERP conceitos, uso e implantação. São Paulo: Atlas, 2001.

ERDIRIK-DOGAN, M.; GROSSMANN, I. E. Simultaneous planning and scheduling of singlestage multi-product continuous plants with parallel lines. Computers and Chemical

Engineering, v. 32, p. 2664-2683, 2008. DOI: 10.1016/j.compchemeng.2007.07.010

FERNANDES, F. C. F. Identificação dos principais autores em planejamento e controle da produção por meio de um Survey com pesquisadores da área. Gestão \& Produção, v.14 (1), p. 83-95, 2007. http://dx.doi.org/10.1590/S0104-530X2007000100008

FUSCO, J. P. A.; SACOMANO, J. B. Operações e Gestão Estratégica da produção. São Paulo: Arte \& Ciência, 2007.

IP, W. H.; LI, Y.; MAN, K.F.; TANG, K. S. Multi-product planning and scheduling using genetic algorithm approach. Computers \& Industrial Engineering, v. 38, p. 283-296, 2000. doi:10.1016/S0360-8352(00)00044-9

KANNEGIESSER, M.; GÜNTHER, H. O.; BEEK, P. V.; GRUNOW, M.; HABLA, C. Value chain management for commodities: a case study from the chemical industry. OR Spectrum, v. 31, p. 63-93, 2009. DOI 10.1007/s00291-008-0124-9

KAPLAN, U.; TURKAY, M.; KARASOZEN, B.; BIEGLER, L. T. Optimization of supply chain systems with price elasticity of demand. INFORMS Journal on Computing, p. 1-12, 2010. http://dx.doi.org/10.1287/ijoc.1100.0421

KIM, Y.; BALLARD, G. Management thinking in the earned value method system and the last planner system. Journal Management Engineering, v. 26, p. 223-229, 2010.

http://dx.doi.org/10.1061/(ASCE)ME.1943-5479.0000026 
MULA, J.; POLER, R.; GARCÍA-SABATER, J.P.; LARIO, F.C. Models for production planning under uncertainly: a review. International Journal of Production Economics, v. 103 (1), p. 271-285, 2006. DOI 10.1016/j.ijpe.2005.09.001

OLIVEIRA, R. C. F. Gerenciamento de Projetos e aplicação da Análise de Valor Agregado em Grandes Projetos. Dissertação (Mestrado) - USP. São Paulo, 2003. PELLEGRINI, F. R.; FOGLIATTO, F. S. Passos para implantação de sistemas de previsão de demanda - técnicas e estudo de caso. Produção, v. 11 (1), 2001. http://dx.doi.org/10.1590/S0103-65132001000100004

ROCHA, D. Fundamentos técnicos da produção. São Paulo: Makron Books, 1996.

RUBIO, A. L. Planejamento e a programação da produção: entendendo os conceitos e técnicas utilizadas nos diversos ambientes de produção, São Paulo: STS, 2002.

SEVERIANO, C. F.; DUNDA, M. F. E.; BATISTA, G. B. Análise das Abordagens sobre Medidas de Produtividade. ENEGEP, 1997.

SILVA FILHO, O. Estratégias Sequenciais Subótimas para Planejamento Agregado da Produção sob Incertezas. Gestão \& Produção, v. 7 (3), p. 247-268, 2000.

https://doaj.org/article/b5c0d2f2551e4be69ee51814892b0a33

SLACK, N.; CHAMBERS, S.; JOHNSTON, R. Administração da produção. 2 ed. São Paulo: Atlas, 2002.

TUBINO, D. F. Manual de planejamento e controle da produção. São Paulo: Atlas, 1997.

VANDEVOORDE, S; VANHOUCKE, M. A comparison of different project duration forecasting methods using earned value metrics. International Journal of Project Management, v. 24, p. 289-302, 2005. DOI:10.1016/j.ijproman.2005.10.004

VIEIRA, G. E; SOARES, M. M. A new multi-objective optimization method for master production scheduling problems based on genetic algorithm. International Journal

Advanced Manufacturing Technology, v. 41, p. 549-567, 2009. DOI: $10.1007 / \mathrm{s} 00170-008-$ $\underline{1481-\mathrm{X}}$

VITNER, G, ROZENES S, SPRAGGET S. Using data envelope analysis to compare project efficiency in a multi-project environment. International Journal of Project Management, v. 24, p. 323-329, 2006. DOI:10.1016/j.ijproman.2005.09.004

XIUTIAN,S.; HOUCAI, S.; TING, W.; CHENG, T. Production planning and pricing policy in a make-to-stock system with uncertain demand subject to machine breakdowns. European Journal of Operational Research, v.238, p. 122-129, 2014. DOI:

10.1016/j.ejor.2014.03.017

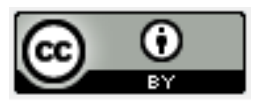

Artigo recebido em 10/04/2016 e aceito para publicação em 08/07/2016

DOI: http://dx.doi.org/10.14488/1676-1901.v17i1.2419 